\title{
In situ measurement of wave attractor induced forces
}

\author{
Alexander M. van Oers ${ }^{1} \mathbb{D} \cdot$ Leo R. M. Maas $^{2} \mathbb{D}$
}

Received: 14 May 2021 / Revised: 6 August 2021 / Accepted: 11 August 2021 / Published online: 22 December 2021

(c) The Author(s) 2021

\begin{abstract}
An underwater force sensor for internal waves is presented. Using this sensor, we measure forces at a location near the surface of a fluid. The sensor performs a point measurement with a high temporal resolution. We perform measurements in a densitystratified fluid contained in a trapezoidal basin. By shaking this basin longitudinally, internal gravity waves are generated. Controlling the frequency with which the basin oscillates, these waves propagate toward a wave attractor whose shape varies from complicated to rectangular. We measure the force exerted by these waves on a plate that is partially submerged into the fluid. We observe the formation and decay of wave attractors. When a surface reflection of a wave attractor is near our sensor we measure (relatively) strong forces. We confirm our findings with simulations. We observe an asymmetry in the direction of the force. This asymmetry leads to a net force and could imply the driving of a mean flow.
\end{abstract}

\section{Graphical Abstract}

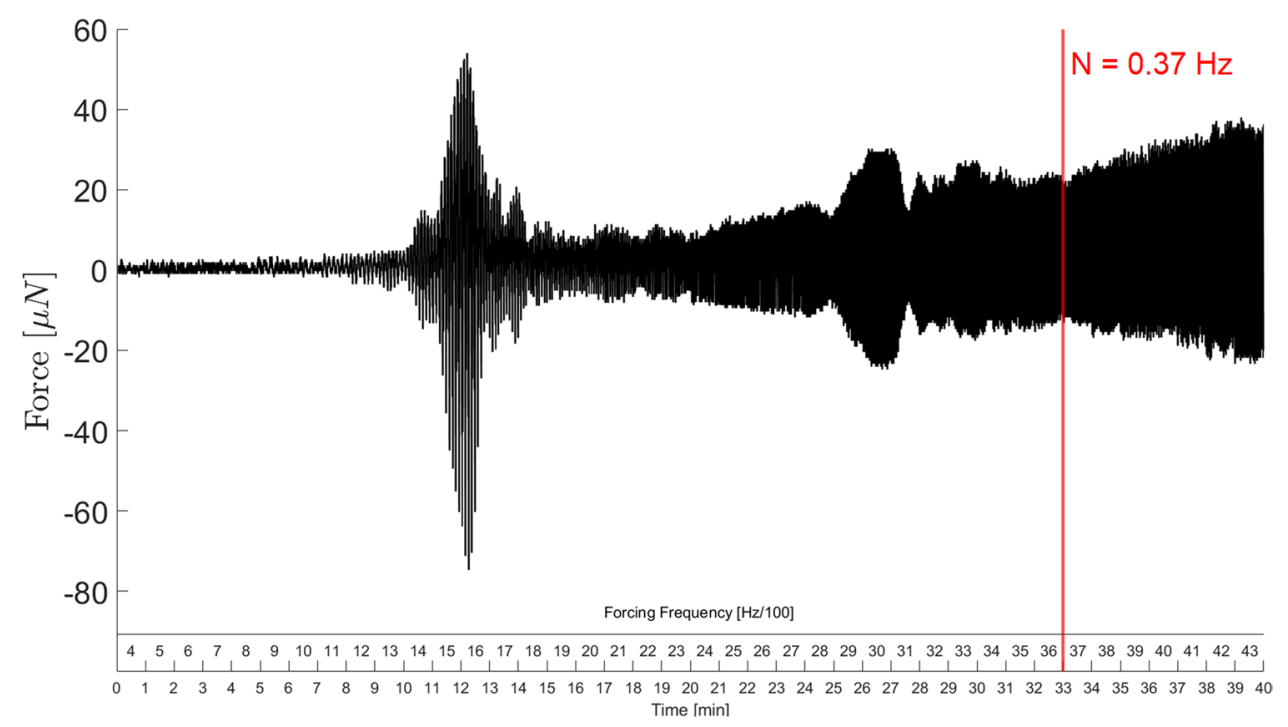

Internal waves only exist when the fluid in which they propagate is stratified in density (internal gravity waves) or angular momentum (inertial waves). These waves propagate

Alexander M. van Oers

AM.v.Oers@mindef.nl

1 Faculty of Military Science, Netherlands Defence Academy, Den Helder, The Netherlands

2 Institute for Marine and Atmospheric Research Utrecht (IMAU), Utrecht University, Princetonplein 5, 3584 CC Utrecht, The Netherlands obliquely relative to the stratifying direction. The angle of propagation is fixed by the wave's frequency and the stratification. Waves of given frequency (e.g., tides) preserve this inclination when reflecting from any boundary, also when reflecting from a sloping boundary. The consequence of this constraint is that for almost any shape of basin, these waves are focused onto a limit orbit, called a wave attractor (Maas 1997).

The existence of internal and inertial wave attractors was shown in experiments (Maas and Lam 1995; Maas 2005). In 
Maas and Lam (1995), perturbations were generated using parametric resonance with vertical oscillations of a tank. Later, experiments generated wave attractors in density stratified fluid by horizontal oscillations of a tank (Hazewinkel et al. 2008, 2011a). Internal wave attractors were shown to persist despite basins having non-uniform stratification, small-scale boundary corrugations (Hazewinkel 2010) or being forced non-centrally in a three-dimensional (paraboloidal) domain (Hazewinkel et al. 2011a). In Gostiaux (2007), an internal wave generator is discussed which allows more energy to be inserted into the system. This led to the discovery of a parametric subharmonic instability (PSI) via a triadic resonance (Scolan et al. 2013).

Two optical non-intrusive measurement techniques that are often used to study internal gravity waves are Synthetic Schlieren (Dalziel et al. 2000) and particle image velocimetry [PIV, e.g., Maas (2005)]. See, e.g., Hazewinkel (2008, 2010) for 2D observations; and Manders and Maas (2003); Hazewinkel (2011b); Pillet (2018) for 3D observations. In Synthetic Schlieren, density perturbations caused by internal gravity waves change the path of light rays through the fluid. These changing light rays are tracked using a background reference. Using digital image correlation, Synthetic Schlieren relates the apparent changes in the still reference back to the gradients of the density perturbations. This allows a full cross-sectionally averaged view of the 2D field of density gradients. In PIV, motions are visualized by illuminating particles within a laser sheet. These particles form a dot pattern similar to Synthetic Schlieren. By following the motion of these patterns, the local velocity can be inferred. PIV gives velocity fields in a plane, yielding a snapshot of the flow. By rapidly changing the plane of measurement, a $3 \mathrm{D}$ velocity field can be determined.

We are interested in the behavior of wave attractors near the reflection locations, in particular their surface reflections. These reflection locations can be the source of interesting dynamics, such as triad interactions (Dauxois 2018), and can leave an impact traceable from satellite altimeter observations (da Silva 2012). Internal tides reflecting on continental slopes can deposit sediments and possibly erode the sediment surface (Cacchione et al. 2002). Because an attractor can experience strong gradients, it can be a challenge to perform accurate measurements inside these regions. To investigate the behavior of a wave attractor near the surface of a fluid, we have designed a new sensor. This sensor measures the horizontal force at one location near the surface. The sensor consists of a small vertical plate that deflects due to the movement of the fluid. This deflection is measured and related to the force exerted by the ambient pressure difference.

Many studies have been performed on the generation of internal gravity waves by oscillating objects in stratified fluids. One of the first experimental studies on internal gravity waves was (Mowbray and Rarity 1967). Here, a classical Schlieren technique was used to measure the internal gravity waves generated by a circular cylinder oscillating horizontally or moving a flat strip horizontally. Later, studies focused on the generation of internal gravity waves by oscillating spheres (e.g., Voisin et al. (2011); Brouzet (2017) and references therein). With our sensor, we have gone the opposite route: instead of oscillating an object and studying the generated internal waves, we generate internal waves and study the response of the object. We use a small, thin flat plate as the object. By tracking the movement of the plate very accurately, we can measure the response of the plate caused by the internal waves.

In Sect. 1, we discuss the design of our sensor. We start with the deflection of a vertical plate due to a force normal to the plate and the measurement of this deflection. This setup is calibrated and several control experiments are performed. We model the fluid-solid interaction to relate the measured forces to horizontal velocities. In Sect. 2, we discuss the experimental setup, the generation of wave attractors and the use of our new sensor. In Sect. 3, we show the measurements from our new sensor. We use a range of frequencies, including those for which wave attractors appear. Individual and continuous measurements are performed. We reproduce our experiments with simulations. In Sect. 4, we give some remarks on our sensor and the observations on wave attractors. We make some suggestions for improvements on our sensor.

\section{Design of a force measurement}

The main part of our sensor is a small vertical plate, the dimensions of which are shown in Fig. 1. The main design consideration for our sensor is that we want to perform force measurements in the flow without disturbing the flow. To prevent too much flow disturbance from our sensor, we use a plate with a small width. To achieve a large sensitivity of the sensor, we use a plate that is very thin. The plate is clamped above the fluid, penetrates the surface of the fluid and has a free end in the fluid. A force is exerted on the submerged part of the plate due to fluid movement. The plate deflects due to a transverse force and this deflection is measured above the surface of the fluid by a laser displacement sensor. Figure 2 shows a schematic of this process. In the coming sections, we explain the design further.

\subsection{Relation between force and deflection of a flat plate}

We model the force exerted by the fluid as a point force acting $5 \mathrm{~mm}$ from the tip of the plate, at the green dot in Fig. 1 . For one fixed end at $s=d$ and a small force $F_{x}$ applied at 


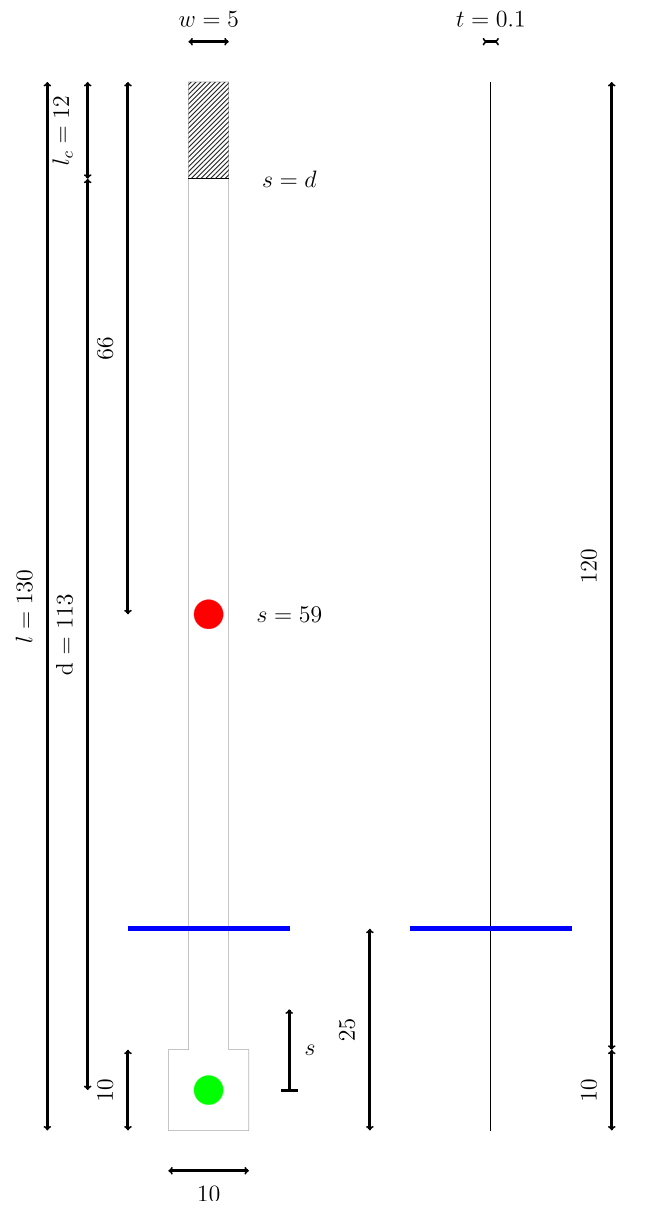

Fig. 1 Front (left) and side (right) views of the plate with dimensions in $\mathrm{mm}$. The plate is clamped at the top (hashed) with a clamp length $l_{c}=12 \mathrm{~mm}$ and has a free end at the bottom. The laser displacement sensor measures the deflection of the plate at the red dot. The weights in the calibration are placed at the green dot. The blue line is the surface level of the fluid

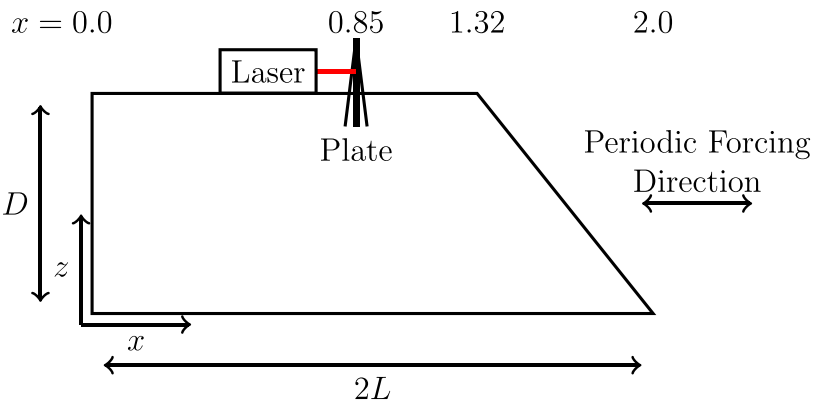

Fig. 2 Schematic overview of the experiment. The plate is clamped from above while the free end is located inside the fluid. The laser displacement sensor measures the distance (shown in red) to the plate above the fluid

the free end of the plate at $s=0$, the deflection $\delta$ along the length of the plate is
$\delta(s)=\frac{F_{x}}{6 E I}\left(s^{3}-3 d^{2} s+2 d^{3}\right)$,

where $0 \leq s \leq d$ is the coordinate along the length of the plate, $d$ the effective length $\left(d=l-l_{c}-5 \mathrm{~mm}=113 \mathrm{~mm}\right.$, with $l$ the total length of the plate and $l_{c}$ the length of the clamp), $E$ the Young's modulus of the material of the plate and $I$ the area moment of inertia of the cross section of the beam (in the $x y$-plane). At every location $s$, the deflection $\delta$ is linear with the applied force $F_{x}$. We want to maximize the deflection of the beam for a given force. We achieve this by minimizing the area moment of inertia I. For a rectangle $I=w t^{3} / 12$, where $w$ is the width and $t$ the thickness. Choosing a small thickness of the plate ensures a very small moment of inertia. A very thin plate has a low resistance to bending when exerting a force perpendicular to the plate.

Figure 1 shows the dimensions of the plate. We use a plate of stainless steel with a thickness of $0.1 \mathrm{~mm}$. Table 1 lists further properties of the plate.

\subsection{Measuring deflections}

When a flow exerts a force on the plate, the plate deflects according to (1). To measure the deflections, we use a laser that measures the distance to an object: a point (above the surface of the fluid) on the plate. This is a Micro-epsilon non-contact laser displacement sensor (optoNCDT1402-10). The optoNCDT 1402-10 laser has a resolution of $\Delta \delta=1 \mu \mathrm{m}$ and measures the deflection at $s=59 \mathrm{~mm}$. The smallest change in force the sensor can measure is $0.6 \mu \mathrm{N}$.

\subsection{Calibration}

The experimental setup consisting of the plate and the laser is calibrated. We place the plate horizontally and the laser above it. The deflection due to self-weight of the plate is constant and is corrected for. By placing small weights (individual staples) on the tip of the plate, the plate deflects. The small weights are placed at the green dot in Fig. 1. The deflection is measured by the laser. We relate the output signal (voltage) to the force exerted on the tip of the plate. Figure 3 shows that the relation between force and output voltage is linear.

\subsection{Control cases}

To test the force sensor, we perform a series of control experiments. First, we test the sensor without any water. Figure $4 \mathrm{a}$ shows a measurement without water. A weak, but nonzero, signal is measured. The frequency of the measured signal corresponds to the forcing frequency. Since the force sensor is placed on top of the tank and the entire tank is oscillated horizontally, the sensor is moving back and forth. The upper 


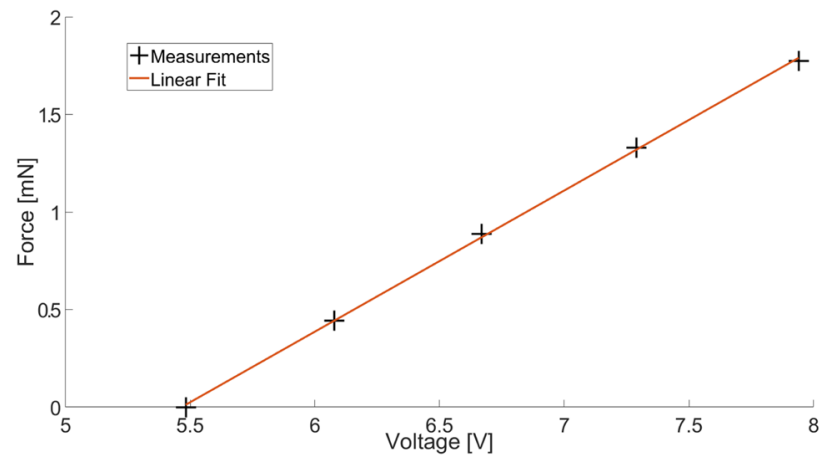

Fig. 3 The calibration consists of five different measurements. For each measurement, a different weight is placed on the tip of the plate

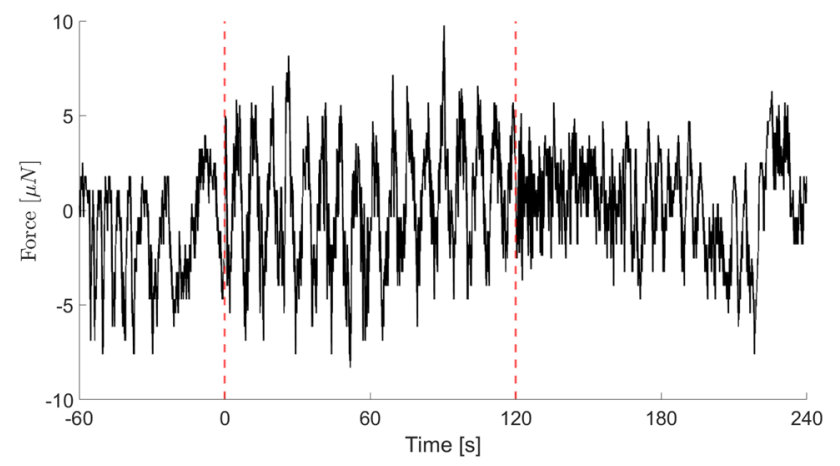

(a) Measurement without water.

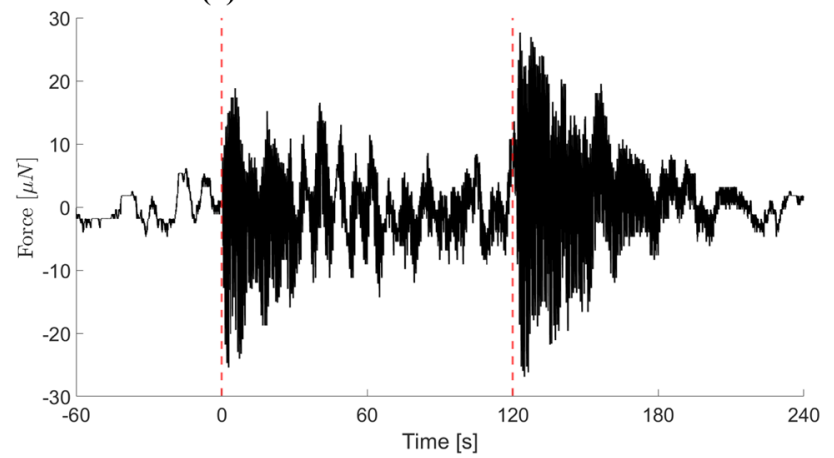

(b) Measurement in homogeneous water.

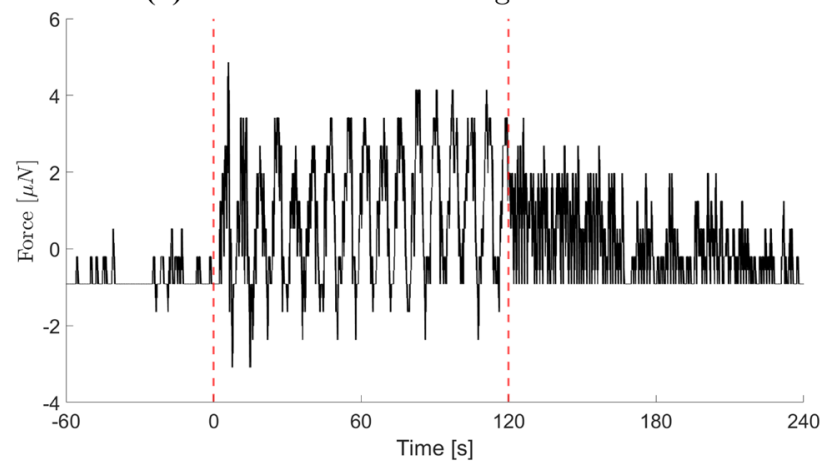

(c) Measurement in homogeneous water with a covered surface.

Fig. 4 Control cases. Dashed lines indicate start and finish of the forcing. Forcing frequency $\omega=0.14 \mathrm{~Hz}$ part of the plate is clamped and moves with the laser and tank. The lower part of the plate is free and displays some inertia. Since the plate is very thin it deforms easily. The motion of the lower end of the plate lags the motion of the upper part and this lag is recorded by the laser. With increase in forcing frequency, this lag becomes larger and the amplitude of the oscillation recorded by the laser increases. The force increases with increase in forcing frequency.

Second, we test the sensor in non-stratified (uniform density) water. Figure $4 \mathrm{~b}$ shows a measurement with water. A strong signal is observed, especially when turning the forcing on or off. The dominant frequencies are those corresponding to the natural frequencies of surface waves in a rectangular container (Ibrahim 2005), which are much higher, $\mathcal{O}(1 \mathrm{~Hz})$, than the forcing frequency $\omega=0.14 \mathrm{~Hz}$. For a trapezoidal domain, the length of the tank at the surface determines the frequencies. No increase in force with forcing frequency is observed. Effectively, we are measuring the effect of surface waves on our sensor. With such a strong signal from surface waves, the effects of internal gravity waves are hard to measure.

Third, we test the sensor in non-stratified water while covering the surface. A small hole exists through which the plate enters the fluid. The hole is just large enough to allow the plate through while not obstructing the movement of the plate. On top of the cover, we place the laser measuring the deflections of the plate. Figure $4 \mathrm{c}$ shows a measurement with a covered surface. The signal is weak, weaker than in the case of a free surface and even weaker than in the case of no water. The water adds an extra damping to the system. No sudden increase in signal when turning the forcing on or off is present. The forcing frequency is the only observed frequency. The force again increases with increase in forcing frequency. The cover successfully removes surface waves from the system.

Last, we perform measurements in stratified water in a rectangular domain. We perform a 'continuous' measurement in which we perform a frequency sweep. Every minute the forcing frequency is increased by $0.01 \mathrm{~Hz}$. Figure 5 shows one such measurement. Independent of the location of the sensor, one resonance frequency dominates. This frequency corresponds to the natural frequency for internal gravity waves in rectangular domains (Maas 2001). No asymmetry in the force is observed. Outside of the resonance frequency, the force increases with increase in forcing frequency.

From these control measurements, we conclude that (1) covering the surface of a fluid effectively damps surface waves and (2) an unwanted background signal, related to inertia of the sensor's loose end, is present. This background signal increases with increase in frequency. 
Fig. 5 Continuous measurement in stratified water in a rectangular domain. The buoyancy frequency was $N=0.37 \mathrm{~Hz}=2.3 \frac{\mathrm{rad}}{\mathrm{a}}$. Spikes reveal the change in forcing frequency

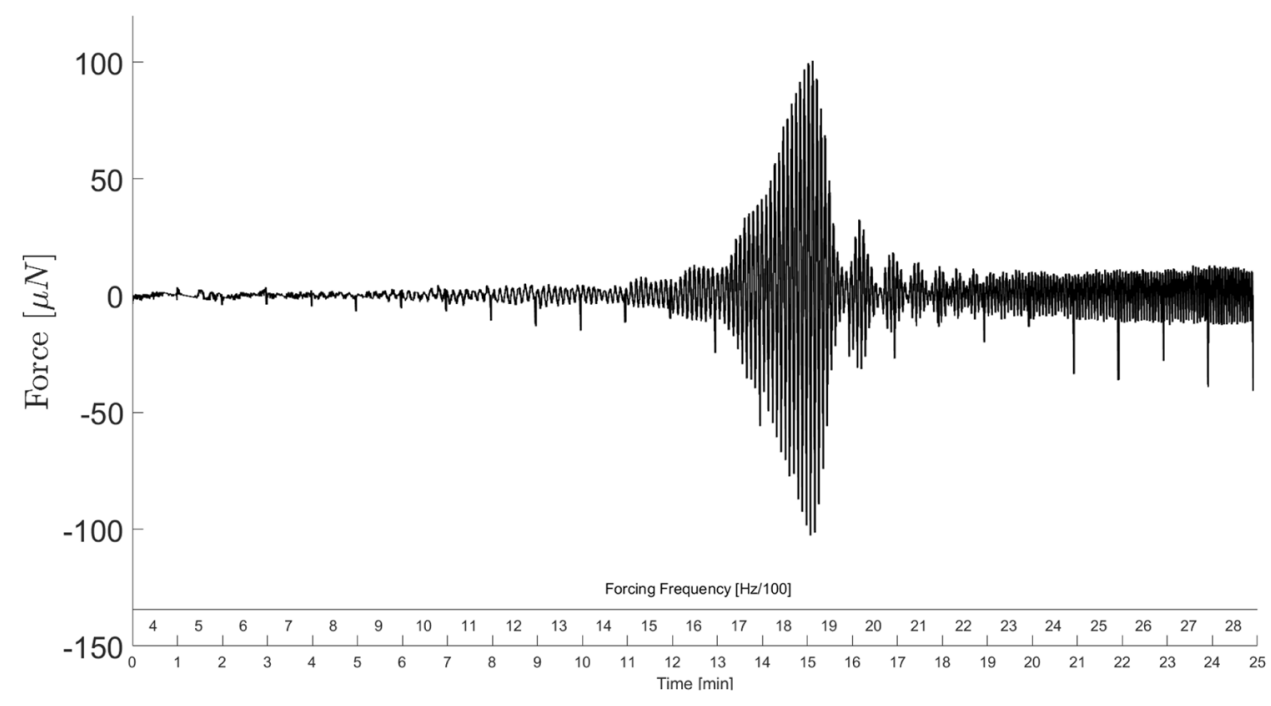

\subsection{Relation between flow and the force on a flat plate}

We place the plate perpendicular to a background flow. The force exerted by a flow on a flat plate has been studied extensively [e.g., Grift (2019)]. We use an empirical relation, called the Morrison equation, where the drag force $F_{x}$ is

$F_{x}(t)=F_{C D}+F_{v m}=\frac{1}{2} C_{D} \rho u|u| S+\left(m_{p}+m_{a}\right) \frac{d u}{d t}$,

where $F_{C D}$ is the steady-phase drag force, $F_{v m}$ the drag force due to the accelerating fluid, $\rho$ is the density of the fluid, $u$ the horizontal velocity of the fluid, $S$ the surface area of the submerged part of the plate and $C_{D}$ the drag coefficient. The added mass is the displaced mass times an added mass coefficient $C_{A}: m_{a}=\rho S t C_{A}$, where $t$ is the thickness of the plate.

Since (2) is an empirical formula, we turn to experiments to determine the hydrodynamic coefficients, $C_{A}$ and $C_{D}$. In Tian (2017), a series of experiments on oscillating flat plates were performed. Rectangular plates with different aspect ratios were also studied. Since the plates were periodically forced, the reported drag coefficients $C_{D}$ and added mass coefficients $C_{A}$ were averaged over the forcing period. Tian (2017) report the results as a function of the Keulegan-Carpenter number $K C=u T / D$, with $u$ the maximum (horizontal) velocity of the oscillation, $T$ the period of the oscillation and $D$ a characteristic dimension of the plate. We estimate $u$ from our simulations. For a typical measurement in our experiments, the Keulegan-Carpenter number is $K C=3.3$. This is just outside the range of the experiments of (Tian 2017). We use linear extrapolation to determine the coefficients. We find a drag coefficient of $C_{D}=3.6$ and an added mass coefficient of $C_{A}=90$. The added mass becomes $m_{a}=1.58 \mathrm{~g}$. The mass of the submerged part of the plate is $m_{p}=0.14 \mathrm{~g}$. The added mass $m_{a}$ is about 11 times as large as the mass of the plate $m_{p}$.

Using the linear non-viscous horizontal momentum equation, the time derivative $\frac{d u}{d t}=-\frac{1}{\rho} \frac{\partial p}{\partial x}$. Substituting this in (2), we obtain

$F_{x}=\frac{1}{2} C_{D} \rho u|u| S-\left(m_{p}+m_{a}\right) \frac{1}{\rho} \frac{\partial p}{\partial x}$.

The force acting on the plate consists of a term proportional to the square of the velocity and a term proportional to the horizontal pressure gradient. Assuming a plane wave with a periodic signal with frequency $\omega$, time derivative $\frac{d u}{d t}=i \omega u$. Then, the second term in (3) is proportional to the product of horizontal velocity and frequency.

\subsection{Interpretation as a ribbon microphone}

A ribbon microphone is an instrument designed to sense pressure waves. It has a metal sheet called a ribbon or diaphragm, both sides of which are exposed to the medium around it. The diaphragm is clamped on both sides and placed in a magnetic field. The diaphragm acts as a conductor: movements of the diaphragm cause a potential difference. The exposure on both sides makes ribbon microphones sensitive to pressure differences instead of pressure itself. If such a ribbon microphone is placed in a pressure field, the force $F_{\text {ribbon }}$ acting to move the diaphragm is Beranek and Mellow (2019)

$F_{\text {ribbon }}=-S \frac{\partial p}{\partial x} \Delta l \sin \alpha$,

where $S$ is the effective surface area of the diaphragm, $\partial p / \partial x \sin \alpha$ the component of the $x$-gradient of the pressure acting across the faces of the diaphragm and $\alpha$ the angle of 
attack of the diaphragm with respect to the flow. $\Delta l$ is the effective path length: the difference in pressure between the two sides of the ribbon is the same as that in a pressure field between two points in space separated by this distance. It is this difference in pressure due to the difference in phase between the front and back that actuates the ribbon in the ribbon microphone.

Using the horizontal momentum equation for a fluid and $\alpha=90^{\circ}$ in (4) and comparing to the second term of (2), we observe that the two terms are similar.

Our sensor has the same working principle as a ribbon microphone. Waves travel past a plate, which deflects due to a force exerted by a pressure differential. These deflections are measured to approximate the local pressure gradient of the fluid. A difference is that we measure the deflection with a laser displacement sensor, while in ribbon microphones, the deflection is measured by potential differences. Just like ribbon microphones, our sensor is proportional to the frequency of the wave.

\section{Experimental setup}

\subsection{Generating internal gravity waves and wave attractors}

For the experiment, we use a narrow rectangular tank. The tank is filled with a stratification, in which the density decreases linearly upwards. The background density consists of a constant part $\rho^{\star}$ and a much smaller (by a factor 100) spatially-varying part $\rho_{0}(z)$. This stratification is built using the so-called double-bucket technique. After filling, an inclined side wall is placed in the tank. This breaks the symmetry of the domain. The entire tank is positioned on a table with wheels that can oscillate horizontally with an amplitude of $2 \mathrm{~cm}$ and a forcing frequency $\omega$. The generated internal waves have a frequency equal to the forcing frequency.

The dispersion relation $\omega=N \cos \theta$ relates the frequency $\omega$ of the internal waves to the buoyancy frequency $N=\sqrt{-g / \rho^{\star} d \rho_{0}(z) / d z}$. For constant buoyancy frequency $N$ and constant frequency $\omega$, internal waves propagate energy in a straight line inclined at a constant angle $\pm \theta$. By periodically forcing the fluid at different frequencies $\omega$ and measuring the angle $\theta$ at which internal gravity waves propagate, the buoyancy frequency $N$ is determined: $N=0.37 \mathrm{~Hz}=2.3 \frac{\mathrm{rad}}{\mathrm{s}}$. After completing the measurements, this procedure is repeated to confirm the stratification has not changed.

For certain frequency bands, $(m, n)$ wave attractors appear, where integers $m$ and $n$ indicate the amount of surface and sidewall reflections of the limit cycle, respectively. Using the geometric description from Maas (1997), we construct a Poincaré plot for the experimental setup: see Fig. 6. For each frequency, a simulation is done and

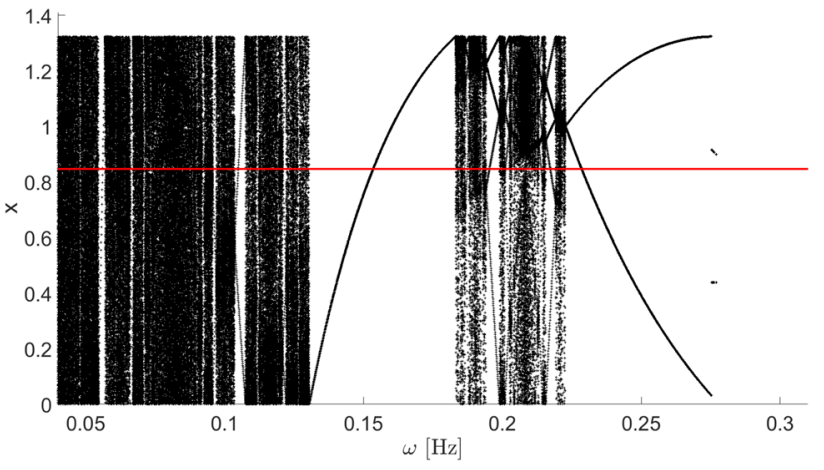

Fig. 6 A Poincaré plot of the experimental setup. The red line indicates the measurement location. The dimensionless variable $x$ indicates the position of the reflection point at the surface of the fluid scaled with halfwidth $L$, with $x=0$ being the leftmost point and $x=2$ the rightmost point, see the top of Fig. 2

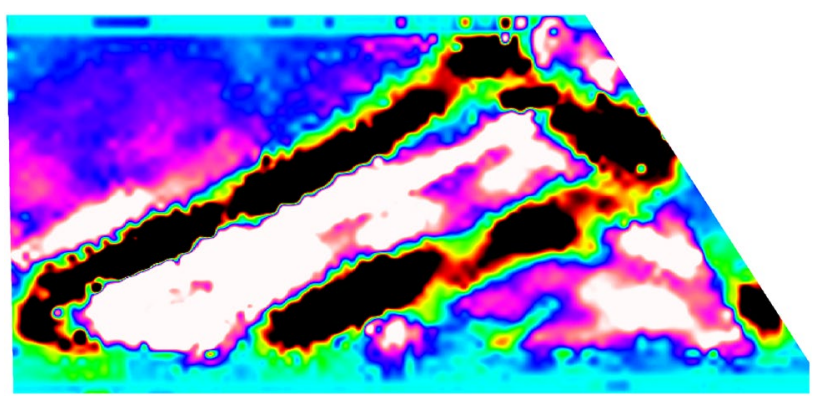

Fig. 7 A qualitative view of the vertical gradient of the density perturbation of a wave attractor in the trapezoidal domain of our experiments, visualized using Synthetic Schlieren. The colors indicate the strength of the gradients: black a strong positive gradient and white a strong negative gradient. This $(1,1)$ wave attractor has one reflection point at the surface and one at its vertical side wall. The bottom of the domain was just out of view of the camera. The forcing frequency was $0.16 \mathrm{~Hz}$

the locations of the surface reflections are shown. When, for a given frequency, there are many surface reflections (large $m$ and $n$ ), internal gravity waves are focused onto a high-period, 'complicated', limit cycle or an ergodic orbit (Lenci et al. 2021), both involving many boundary reflections and subject to viscous damping. When there is one (or a few) surface reflection(s), (small $m$ and $n$ ) internal gravity waves are focused onto an excitable limit cycle and wave attractors can appear. Figure 6 shows there is a frequency band around $0.15 \mathrm{~Hz}$ for which $(1,1)$ wave attractors appear and another frequency band around $0.25 \mathrm{~Hz}$ for which $(2,1)$ wave attractors appear. We use Synthetic Schlieren (Dalziel et al. 2000) to confirm the existence of wave attractors in these frequency bands. Figure 7 shows a wave attractor generated in our experiments. It has amplified perturbations located in the vicinity of the theoretically predicted rectangle (roughly identical with the middle of the black regions). 
Phase propagation (not shown) is in a direction perpendicular to each of the four branches and indicates the internal wave propagates its energy in a counterclockwise fashion around this rectangle.

\subsection{Using the force sensor}

At the surface, we insert the plate in the fluid near the middle of the domain. We place the plate parallel to the direction of gravity, parallel to the $z$-axis in Fig. 2. We assume internal gravity waves propagate in the xz-plane and no movement exists in the $y$-direction. Then, the angle of attack $\alpha$ of the plate with respect to the flow is $90^{\circ}$.

The location and properties of our sensor (and our experiment) are listed in Table 1. Since the plate is clamped above the fluid and the free end is below the surface of the fluid, the surface area $S$ that experiences a force from the fluid is only that part of the plate that is submerged.

The sign of the force $F$ relative to the horizontal velocity $u$ is determined by the position of the laser. In Fig. 2 the laser is positioned to the left of the plate. When a positive horizontal velocity exists (around the location of the plate), the flow is from left to right in Fig. 2. The plate will deflect to the right, with the flow. The distance between the laser and the plate will increase: a positive force is measured.

\section{Results}

We performed measurements for a fixed domain, buoyancy frequency and measurement location. Two types of measurements were performed: individual and continuous. In individual measurements, we keep the forcing frequency constant during the experiments. The forcing is turned on for five minutes and then off. Each measurement is started from rest. This allows us to see the build-up and decay of wave attractors. In continuous measurements, the forcing is turned on and the forcing frequency is changed stepwise without stopping the forcing. This allows a sweep over the entire frequency range.

\subsection{Individual measurements}

For each individual measurement, we changed the frequency $\omega$. Figures 8, 9, 10 and 11 show measurement results for different frequencies. The first dashed vertical line indicates the start of the forcing ( $t=0 \mathrm{~s})$ and the second dashed vertical line the end of the forcing $(t=300 \mathrm{~s})$. Figure 6 shows for which frequencies we expect to measure signals from large-scale (small $m$ and $n$ ) wave attractors: when the surface reflection is near the location of our sensor.

Figure 8 shows the measured force for a frequency of $0.04 \mathrm{~Hz}$. This is the lowest frequency we measure and it
Table 1 Parameters of experiment

\begin{tabular}{lll}
\hline Parameter & Symbol & Value \\
\hline Water tank & & \\
Forcing frequency & $\omega$ & $0.04-0.26 \mathrm{~Hz}$ \\
Amplitude forcing & $N$ & $2 \mathrm{~cm}$ \\
Buoyancy frequency & D & $0.37 \mathrm{~Hz}=2.3 \frac{\mathrm{rad}}{\mathrm{s}}$ \\
Height & & $24.5 \mathrm{~cm}$ \\
Length at surface & $2 L$ & $43 \mathrm{~cm}$ \\
Length at bottom & & $65 \mathrm{~cm}$ \\
Width & & $13.5 \mathrm{~cm}$ \\
Angle of sloping wall & & $42^{\circ}$ \\
Duration forcing stage & & $300 \mathrm{~s}$ \\
Duration decay stage & & $120 \mathrm{~s}$ \\
Plate & & \\
Thickness & $t$ & $0.1 \mathrm{~mm}$ \\
Length & $l$ & $130 \mathrm{~mm}$ \\
Width & $w$ & $5-10 \mathrm{~mm}$ \\
Clamp length & $l c$ & $12 \mathrm{~mm}$ \\
Depth submerged & & $25 \mathrm{~mm}$ \\
Surface area submerged & $S$ & $1.75 \times 10^{-4} \mathrm{~m}^{2}$ \\
Density & & $8 \times 10^{3} \mathrm{~kg} \mathrm{~m}^{-3}$ \\
Young's modulus & $E$ & $200 \mathrm{GPa}$ \\
Area moment of inertia & $I$ & $4.2 \times 10^{-4} \mathrm{~mm}^{-4}$ \\
Horizontal location & & $27.5 \mathrm{~cm}$ \\
Dimensionless location & $x$ & 0.85 \\
\hline
\end{tabular}

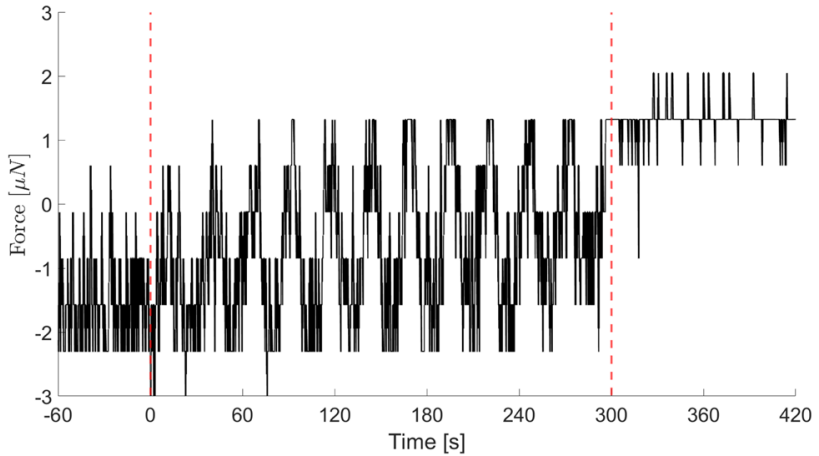

Fig. 8 The measured force for a forcing frequency of $0.04 \mathrm{~Hz}$. The signal is close to the resolution of $0.6 \mu \mathrm{N}$. Dashed lines indicate start and finish of the forcing. The amplitude of the measured force is $1.8 \mu \mathrm{N}$ resulting in a velocity amplitude of $1.0 \mathrm{~mm} \mathrm{~s}^{-1}$

gives the weakest signal. The signal is close to the resolution of $0.6 \mu \mathrm{N}$. The position of the plate before and after the experiment shifted slightly. This is due to the moment at which the tank starts and stops in its oscillation cycle. We see a periodic signal with a frequency corresponding to the forcing frequency. Internal gravity waves propagate through the fluid but no wave attractor forms. 


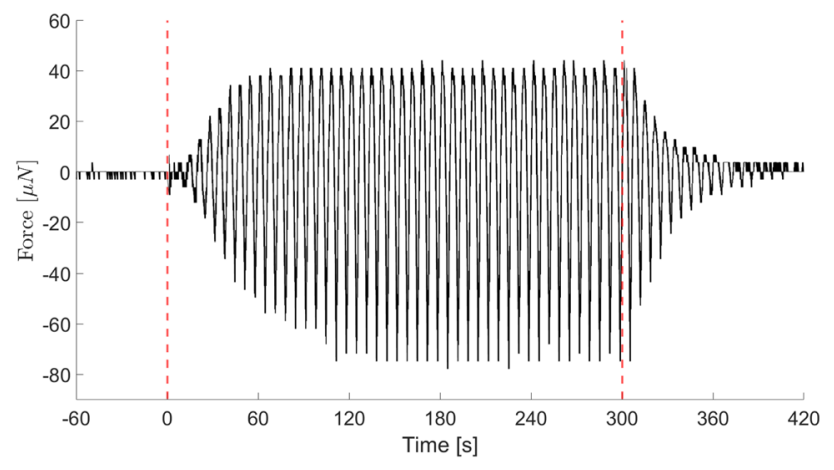

Fig. 9 The measured force for a forcing frequency of $0.15 \mathrm{~Hz}$. This frequency corresponds to the strongest wave attractor in our experiments. Dashed lines indicate start and finish of the forcing. During the periodic steady state, the mean force is $-17 \mu \mathrm{N}$ and the amplitude of the periodic signal is $58 \mu \mathrm{N}$. This implies a mean velocity of $-6 \mathrm{~mm} \mathrm{~s}^{-1}$ and an amplitude of the periodic velocity of $14 \mathrm{~mm} \mathrm{~s}^{-1}$

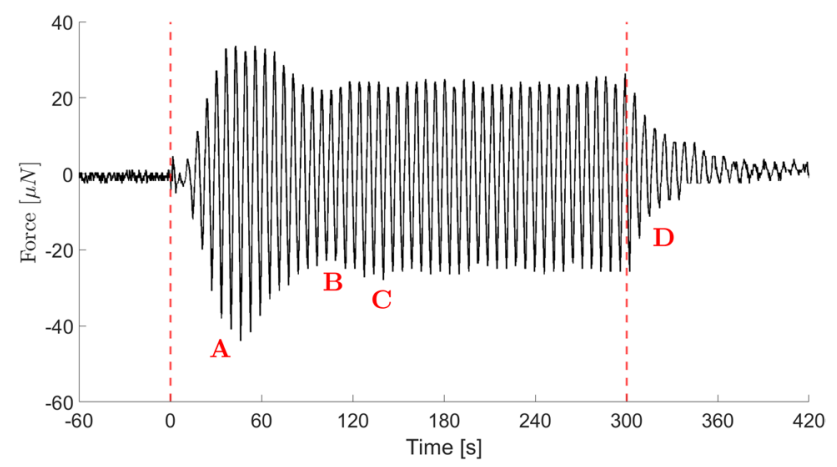

Fig. 10 The measured force for a forcing frequency of $0.16 \mathrm{~Hz}$. This frequency corresponds to a wave attractor. Dashed lines indicate start and finish of the forcing. After the forcing starts, an overshoot (A) occurs followed by viscous overrelaxation (B) before a periodic steady state is reached (C). After the forcing stops, an exponential decay (D) is observed. During the periodic steady state, the mean force is $-0.87 \mu \mathrm{N}$ and the amplitude of the periodic signal is $27 \mu \mathrm{N}$. This implies a mean velocity of $-0.53 \mathrm{~mm} \mathrm{~s}^{-1}$ and an amplitude of the periodic velocity of $8.4 \mathrm{~mm} \mathrm{~s}^{-1}$

Figure 9 shows the measured force for a frequency of $0.15 \mathrm{~Hz}$. This frequency corresponds to the strongest wave attractor in our experiments: we can see the attractor occur at the inclined wall by the naked eye. We observe a build-up phase and a saturation phase in the form of a periodic steady state. After the forcing is stopped, the signal slowly decays: the wave attractor persists for a while after turning off the shaking. The exponential decay time is $25 s$ or 3.75 wave periods. Figure 10 shows the measured force for a frequency of $0.16 \mathrm{~Hz}$. For this frequency, a weaker $(1,1)$ wave attractor is present. After the build-up phase, we observe an overshoot (also seen in Synthetic Schlieren measurements (Lam and Maas 2008) with a maximum amplitude of the force of $44 \mu \mathrm{N}$ and an overcompensation due to viscous effects

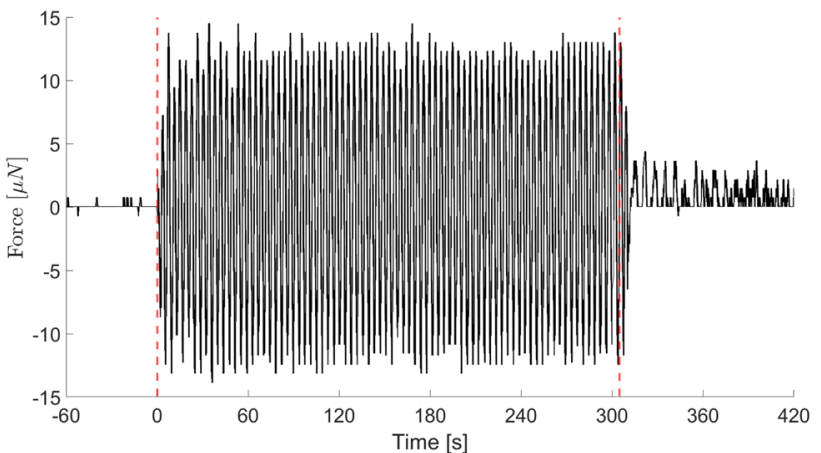

Fig. 11 The measured force for a forcing frequency of $0.26 \mathrm{~Hz}$. Dashed lines indicate start and finish of the forcing. The amplitude of the measured force is $12.3 \mu \mathrm{N}$ resulting in a velocity amplitude of $4.8 \mathrm{~mm} \mathrm{~s}^{-1}$

with an amplitude of $23 \mu \mathrm{N}$ before a periodic steady state is reached with an amplitude of $27 \mu \mathrm{N}$. For some frequencies where wave attractors appear this overshoot is observed, but not for all frequencies.

The force measurements in Figs. 9 and 10 show an asymmetry in the direction of the force. Negative forces are larger than positive forces. A negative force is in the negative $x$-direction. The observations made using Synthetic Schlieren show the wave attractors are traversed anti-clockwise. The signal is strongest in the upper right branch of the wave attractor and weakens along the path of the wave attractor due to viscous effects. The focusing reflection and resulting amplification occur at the inclined side wall [e.g., Chalamalla and Sarkar (2016)]. A net force, pointing away from the inclined side wall, is observed. This net force indicates the existence of a mean flow.

For a forcing frequency of $0.15 \mathrm{~Hz}$ this mean force is large: During the periodic steady state, the mean force is $-17 \mu \mathrm{N}$ and the amplitude of the periodic signal is $58 \mu \mathrm{N}$. Although a mean force breaks our assumption of a periodic flow, we still use (3) with a periodic flow to compute the velocities. This force implies a mean (horizontal) velocity of $-6 \mathrm{~mm} \mathrm{~s}^{-1}$ and an amplitude of the periodic (horizontal) velocity of $14 \mathrm{~mm} \mathrm{~s}^{-1}$. The mean flow is almost half of the periodic flow velocity. Such strong mean flows have been observed before (Bordes 2012). For a forcing frequency of $0.16 \mathrm{~Hz}$, the mean force is $-0.87 \mu \mathrm{N}$ and the amplitude of the periodic signal is $27 \mu \mathrm{N}$ during the periodic steady state. This implies a mean velocity of $-0.53 \mathrm{~mm} \mathrm{~s}^{-1}$ and an amplitude of the periodic velocity of $8.4 \mathrm{~mm} \mathrm{~s}^{-1}$. The mean flow is about $5 \%$ of the periodic flow velocity, similar to the results in (Brouzet 2016). The periodic velocity amplitudes for both frequencies are similar to those measured in Hazewinkel et al. (2011a) for comparable experiments.

Figure 11 shows the measured force for a frequency of $0.26 \mathrm{~Hz}$. The $(2,1)$ wave attractor that is present for this 
frequency does not have a reflection point near the plate. When the forcing starts, the signal immediately reaches a periodic steady state. After the forcing is stopped, the signal disappears quickly. Although the frequency increased, the measured force was lower than in Fig. 10. Equation (3) shows us that the horizontal velocity $|u|$ must have been lower (at this measurement location).

\subsection{Simulation}

We turn to simulations to confirm the behavior we find in the experiments. Since our plate is very thin $(510 \mathrm{~mm})$ with respect to the width of the tank $(13.5 \mathrm{~cm})$, we assume the flow does not feel the effects of the plate. So the plate is moved by the flow, but the flow is not influenced by the plate. This simplifies the numerical model. Since the forcing direction is perpendicular to the side walls of the tank and the plate, we assume the flow is two-dimensional.

In van Oers et al. (2017), a numerical model to simulate wave attractors in stratified fluids was developed. This numerical model was constructed in such a way that several conservation laws were obeyed exactly: the numerical model conserved (at least) energy, mass and momentum exactly. Here, we expand their model by including a viscous term and a body forcing term. We solve the non-dimensionalized linear Boussinesq equations

$$
\begin{aligned}
& \frac{\partial \underline{u}}{\partial t}=-\nabla P-\frac{1}{F r^{2}} \rho \underline{z}+\frac{1}{R e} \nabla^{2} \underline{u}+\underline{a}, \\
& \frac{\partial \rho}{\partial t}=F r^{2} N^{2} w \\
& \nabla \cdot \underline{u}=0,
\end{aligned}
$$

where $\underline{u}=(u, w)^{T}$ is the two dimensional velocity field, $\rho$ the scalar perturbation density field, $P$ the mechanical pressure field, $\hat{z}$ is the unit vector in the $z$-direction and $N$ is the buoyancy frequency. The fluid is excited by an external body force $\underline{a}$ per unit mass (Jouve and Ogilvie 2014) with

$\nabla \times \underline{a}=F_{0} \sin (\omega t)$,

where $\omega$ is the forcing frequency and $F_{0}$ the forcing amplitude. The densimetric Froude number Fr and the Reynolds number $R e$ are

$F r=\frac{U}{\sqrt{g \hat{\rho} / \rho^{*} L}}, \quad \operatorname{Re}=\frac{\rho^{*} L U}{\mu}$.

Table 2 lists the different scales used. The numerical model has been implemented in the Portable, Extensible Toolkit for Scientific Computation (PETSc) (Balay et al. 2020; Balay 2019). The mesh is generated using Gmsh (Geuzaine and Remacle 2009).
Table 2 Parameters of the numerical simulation

\begin{tabular}{lll}
\hline Parameter & Symbol & Value \\
\hline Length scale & $L$ & $0.325 \mathrm{~m}$ \\
Time scale & $T$ & $5.76 \mathrm{~s}$ \\
Velocity scale & $U=L / T$ & $0.0565 \mathrm{~ms}^{-1}$ \\
Background density scale & $\rho^{\star}$ & $1 \times 10^{3} \mathrm{~kg} \mathrm{~m}^{-3}$ \\
Perturbation density scale & $\hat{\rho}$ & $1 \mathrm{~kg} \mathrm{~m}^{-3}$ \\
Pressure scale & $\rho^{\star} U^{2}$ & $3.19 \mathrm{~kg} \mathrm{~m}^{-1} \mathrm{~s}^{-2}$ \\
Gravity & $g$ & $9.81 \mathrm{~m} \mathrm{~s}^{-2}$ \\
Dynamic viscosity & $\mu$ & $1 \times 10^{-3} \mathrm{~Pa} \mathrm{~s}^{-2}$ \\
Densimetric Froude number & $F r$ & 1 \\
Reynolds number & $R e$ & $1.8 \times 10^{4}$ \\
Dimensionless buoyancy freq. & $N$ & 2.13 \\
Forcing amplitude & $F_{0}$ & $2.8 \times 10^{-6}$ \\
\hline
\end{tabular}

We repeat our experiments in our simulations. Figure 12 shows a result of our simulation. We take the horizontal velocity and pressure gradient at the location of our sensor (at the green dot in Fig. 1) and use (3) to calculate the forces experienced by the plate in our simulations. Figure 13 shows the force acting on the plate, determined from our simulations.

Comparing the experiments, Fig. 10, with our simulations, Fig. 13, we notice similarities and differences. The overall signal is the same: when the forcing is turned on, a build-up, an overshoot and a viscous overrelaxation occur, after which a periodic steady state is reached. When the forcing is turned off a decay occurs. The amplitude of the periodic steady state in the experiments and simulations are similar. The build-up is very similar. The time until the overshoot occurs is the same: roughly $40 \mathrm{~s}$ after the start of the forcing. A difference is the amplitude of the overshoot: the overshoot amplitude is larger in the experiments. Another difference is the time at which the viscous relaxation is visible: For the experiments, this occurs after roughly two minutes of forcing, while for the simulations, this occurs after $70 \mathrm{~s}$. A third difference is the speed of the decay: the wave attractor decays more rapidly in the simulations than in the experiments. It seems the wave attractor in the simulation can more efficiently dissipate energy than observed experimentally: the decreases in the simulation occur faster (both the viscous overrelaxation and the decay of the wave attractor without forcing). The simulations show no generation of a mean flow, consistent with the linearity of the flow.

The background signal, as shown in Fig. 4c, does not occur in the simulations. The cause of this effect must lie outside the fluid and Morrison's equation. Our hypothesis is that it is caused by the oscillation of entire tank, including the sensor. Figure 11 shows an immediate drop-off in signal when the forcing is stopped. In our simulation for $\omega=0.26 \mathrm{~Hz}$, the signal was weaker. We conclude that the 
Fig. 12 Wave attractor in our simulations. The red line indicates the plate. The figure shows the horizontal velocity after 48 periods with a forcing frequency of $\omega=0.16 \mathrm{~Hz}$

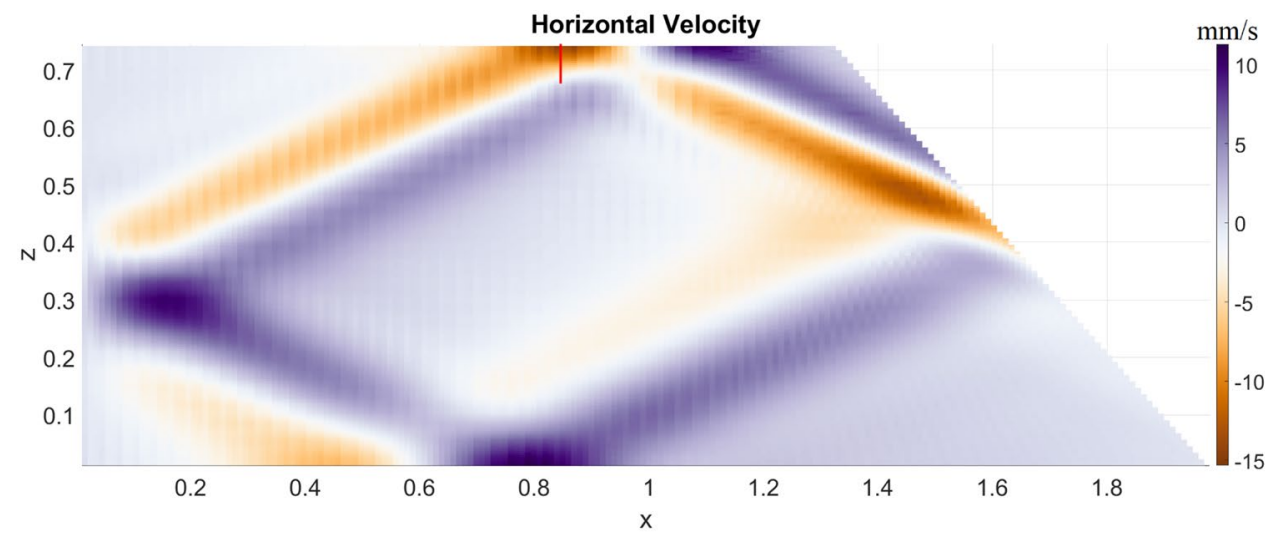

These simulations show that our observations of wave

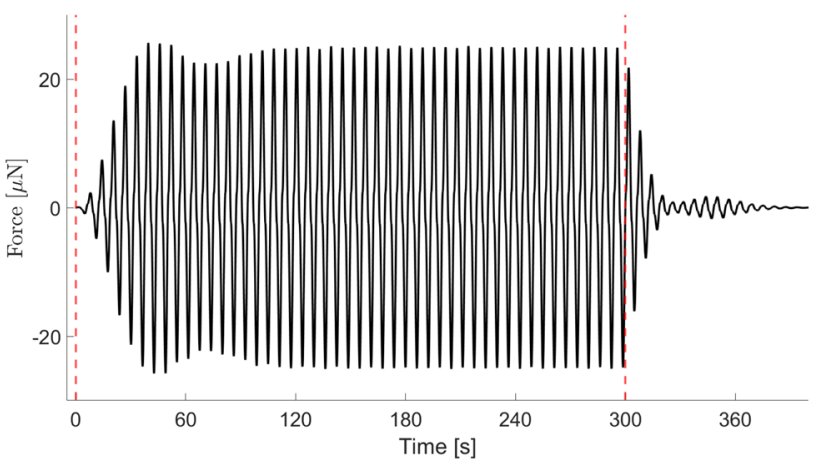

Fig. 13 Force on the plate determined from our simulations for a forcing frequency of $0.16 \mathrm{~Hz}$. The dashed vertical lines indicate start and finish of the forcing. The drag force dominates the inertial force in our simulations

signal in Fig. 11 is dominated by the background signal, related to inertia of the sensor, and does not show a measurement of internal gravity waves. attractors are correct. They also confirm the hydrodynamic coefficients and the use of the Morrison equation.

\subsection{Continuous measurement}

We performed a continuous measurement: every minute the frequency is increased stepwise with steps of $0.01 \mathrm{~Hz}$ starting from $0.04 \mathrm{~Hz}$ and ending at $0.43 \mathrm{~Hz}$. By 'continuously' changing the frequency and never stopping the forcing, a much quicker sweep through a large frequency range is possible. Figure 14 shows this continuous measurement. We make two observations: (1) The large increase in force for a forcing frequency of $0.15-0.16 \mathrm{~Hz}$, corresponding to the range of $(1,1)$ wave attractors that have a surface reflection near the plate. (2) Outside the $(1,1)$ wave attractor band, the imposed force gradually increases with increase in frequency.

Wave attractors are broad-band phenomena, spanning a range of frequencies. The reason this looks like a classical resonance (with one resonance frequency) is that we
Fig. 14 The measured force in a continuous measurement. Every minute the forcing frequency was increased by $0.01 \mathrm{~Hz}$. The peak around $0.15 \mathrm{~Hz}$ corresponds to a $(1,1)$ wave attractor. Outside this $(1,1)$ wave attractor band, the force gradually increases with frequency

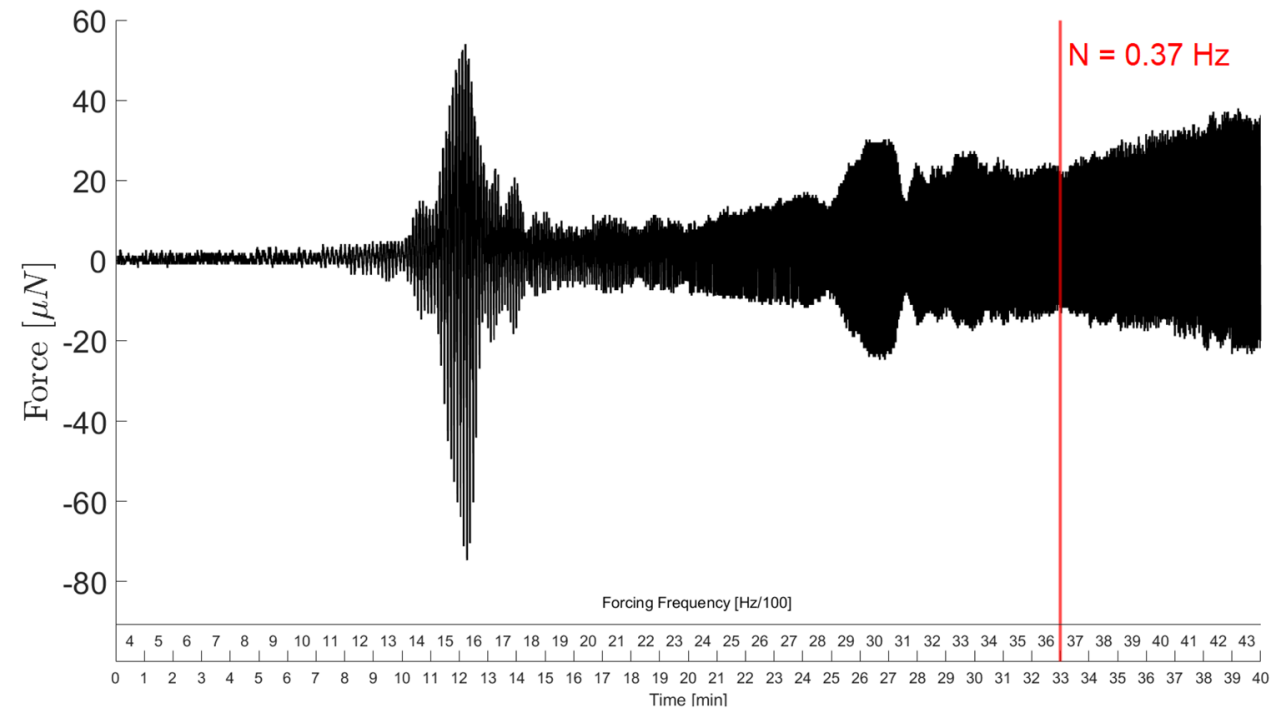


measure at one surface location: the wave attractor that has a surface reflection near the measurement location gives the strongest signal. Further measurements on the $(1,1)$ attractor (not shown here) confirm that forces maximize for slightly different forcing frequencies when putting the force sensor at different x-locations. From Fig. 6, we expect to measure a strong signal when the position of the sensor (red line) and the surface reflection of the wave attractor (black line) intersect. Moving the sensor (along the surface of the tank), we find that the largest signal occurs for slightly different frequencies.

There is an asymmetry in the direction of the force when wave attractors are present. Figures 9 and 10 show that in the first minute the wave attractors are still building up. After two minutes, a periodic steady state is reached. Figure 10 shows that the asymmetry in force is largest during the overshoot, corresponding mostly to the first minute. So, in the continuous measurement we are mostly measuring this overshoot. We also observe that when the frequency changes, the signal collapses as the wave attractor of the old frequency disappears as the wave attractor of the new frequency builds up. For frequencies where no wave attractors appear, this collapse is absent and the signal is uninterrupted.

The unexpected peak around $0.29-0.31 \mathrm{~Hz}$ may indicate that the $(1,1)$ attractor is excited as a subharmonic at half the forcing frequency by parametric excitation as for vertically oscillated tanks (Maas and Lam 1995; Chalamalla and Sarkar 2016; Dauxois 2018).

The absence of a signal for the $(2,1)$ attractor, for a frequency band of approximately $0.22 \mathrm{~Hz}$ to $0.27 \mathrm{~Hz}$, is noticeable. Figure 6 shows that a $(2,1)$ wave attractor exists (at least theoretically). Figure 11 shows that when the surface reflections of the $(2,1)$ wave attractor are not near the measurement location, no signal reminiscent of wave attractors is observed. Since we make steps of $0.01 \mathrm{~Hz}$ for the forcing frequency, we have stepped over the frequency for which the measurement location coincides with the surface reflection position. The forcing frequency $0.22 \mathrm{~Hz}$ is just outside the frequency band for a $(2,1)$ wave attractor. The forcing frequency $0.23 \mathrm{~Hz}$ generates a $(2,1)$ wave attractor with a surface reflection of the left beam which is already to the left of the measurement location. We measured at the wrong location to observe a $(2,1)$ wave attractor. Since the path of a $(2,1)$ wave attractor is longer than that of a $(1,1)$ wave attractor, $(2,1)$ wave attractors are (in general) weaker than $(1,1)$ wave attractors [e.g., Hazewinkel (2010)]. Figures 9 and 10 show that a periodic steady state is reached after two minutes, so the forcing period of 1 minute might be too short for a $(2,1)$ wave attractor to fully form.

\section{Discussion}

We have designed and tested a force sensor for underwater use. This sensor consisted of a plate and a laser displacement sensor. Internal waves exerted a force on the plate, which deformed elastically. A linear relation between force and deflection was found. The force exerted was related to the flow by Morrison's equation. The hydrodynamic coefficients (the drag coefficient and added mass coefficient) were obtained from Tian (2017). Several control measurements were performed. These measurements showed that surface waves dominated the signal of the sensor. Covering the surface removed the surface waves. The control measurements also showed an unwanted background signal.

By periodically forcing a density-stratified fluid, internal gravity waves were excited. Due to the asymmetry of the domain, simple and therefore physically realizable wave attractors appeared for certain frequency bands. Due to the intensification of the wave field near the surface reflections of the wave attractors, we measured (relatively) large forces. From a point measurement, we were able to discern multiple characteristic features of wave attractors: a slow build up, an overshoot, an overcompensation due to viscous effects and saturation in the form of a (periodic) steady-state. After the forcing was stopped, the wave attractor disappeared slowly.

Mean (horizontal) forces were observed for wave attractors. The direction of these mean forces coincided with the direction of travel of the waves along the wave attractor. These mean forces resulted for most $(1,1)$ wave attractors in mean flows of about $5 \%$ of the velocity amplitude, near the surface directed into the horizontal direction into which the internal waves propagated. The generation and direction of a mean flow agrees with observations by Bordes (2012). However, it is at variance with other experimental observations in which particle transport in an inclined internal wave beam seems to be directed toward the wave maker (Horne 2019). Further research needs to elucidate the mean flow generation process and differences in direction. One particularly strong $(1,1)$ wave attractor, see Fig. 9, had a much larger mean force. This large mean force could indicate mean flows that are nearly half of the periodic velocity amplitude. Such a strong mean force could have implications for the horizontal transport of particles in fluids [e.g., Cacchione et al. (2002)].

The downside to our sensor design was a background signal. The control measurements showed that even in measurement with no water and homogeneous water, a periodic signal was measured. The amplitude of this signal increased with the forcing frequency. For large forcing frequencies, e.g., Figure 11, the background signal dominated. Our simulations showed a much smaller amplitude for these large forcing frequencies. The accuracy of our measurements was limited. The cause of this background signal was that the 
entire sensor, including laser and plate, was attached to the tank. The entire tank was oscillated horizontally to generate internal waves. Inertia of the free end of the sensor caused a signal, with a frequency equal to the forcing frequency.

We suggest several improvement to our sensor design:

1. The accuracy of our sensor was limited since it was located on a moving tank, especially at high frequencies. Using an internal wave maker (Gostiaux 2007) would allow the tank to remain stationary and greatly reduce this signal.

2. The material we used for the plate was stainless steel with a Young's modulus of $200 \mathrm{GPa}$. Equation 1 shows that the deflection for a given force decreases with increase in Young's modulus. By using a different material for the plate, like aluminum with a Young's modulus of $70 \mathrm{GPa}$, we can increase the sensitivity of our sensor.

3. We used a plate with a wider part at the bottom. This complicated the analysis. A plate with a uniform thickness of $5 \mathrm{~mm}$ would have behaved similar.

An alternative design of our sensor would be to use a sphere instead of a plate. A lot of theoretical and experimental research has been performed on the drag and added mass coefficients of spheres [e.g., Voisin et al. (2011) and Brouzet (2017)] in stratified fluids. The sphere could be connected by a thin square beam. Two lasers could be employed to measure the forces in both horizontal directions. These kind of measurements (transverse measurements) are particularly hard to obtain from optical methods.

Acknowledgements The authors thank R. de Kat for his suggestion of looking into Ribbon Microphones. The authors thank an anonymous reviewer for the suggestion of using a sphere instead of a plate.

\section{Declaration}

Conflict of interest The authors declare that they have no conflict of interest.

Open Access This article is licensed under a Creative Commons Attribution 4.0 International License, which permits use, sharing, adaptation, distribution and reproduction in any medium or format, as long as you give appropriate credit to the original author(s) and the source, provide a link to the Creative Commons licence, and indicate if changes were made. The images or other third party material in this article are included in the article's Creative Commons licence, unless indicated otherwise in a credit line to the material. If material is not included in the article's Creative Commons licence and your intended use is not permitted by statutory regulation or exceeds the permitted use, you will need to obtain permission directly from the copyright holder. To view a copy of this licence, visit http://creativecommons.org/licenses/by/4.0/.

\section{References}

Balay S et al (2019) PETSc Web page. https://www.mcs.anl.gov/petsc

Balay S et al (2020) PETSc users manual, Argonne National Laboratory, 2020, 754ANL-95/11-Revision 3.14. https://www.mcs.anl. gov/petsc

Beranek L, Mellow T (2019) Acoustics: sound fields. Transducers and vibration. Academic Press, Cambridge

Bordes $G$ et al (2012) Experimental observation of a strong mean ow induced by internal gravity waves. Phys Fluids 24(8):086602086602

Brouzet $C$ et al (2016) Internal wave attractors examined using laboratory experiments and 3D numerical simulations. J Fluid Mech 793:109

Brouzet $C$ et al (2017) Added mass: a complex facet of tidal conversion at finite depth. J Fluid Mech 831:101-127

Cacchione D, Pratson LF, Ogston A (2002) The shaping of continental slopes by internal tides. Science 296(5568):724-727

Chalamalla VK, Sarkar S (2016) PSI in the case of internal wave beam reflection at a uniform slope. J Fluid Mech 789:347-367

Dalziel SB, Hughes GO, Sutherland BR (2000) Whole-field density measurements by 'synthetic schlieren'. Exp Fluids 28(4):322-335

da Silva JC et al (2012) Internal solitary waves in the Red Sea: an unfolding mystery. Oceanography 25(2):96-107

Dauxois T et al (2018) Instabilities of internal gravity wave beams. Ann Rev Fluid Mech 50:131-156

Geuzaine C, Remacle J-F (2009) Gmsh: a 3-D finite element mesh generator with built-in pre-and post-processing facilities. Int J Numer Methods Eng 79(11):1309-1331

Gostiaux L et al (2007) A novel internal waves generator. Exp Fluids 42(1):123-130

Grift EJ et al (2019) Drag force on an accelerating submerged plate. J Fluid Mech 866:369-398

Hazewinkel J et al (2008) Observations on the wavenumber spectrum and evolution of an internal wave attractor. J Fluid Mech 598:373

Hazewinkel J et al (2010) Observations on the robustness of internal wave attractors to perturbations. Phys Fluids 22(10):107102

Hazewinkel J, Grisouard N, Dalziel SB (2011a) Comparison of laboratory and numerically observed scalar fields of an internal wave attractor. Eur J Mech B/Fluids 30(1):51-56

Hazewinkel J, Maas LRM, Dalziel SB (2011b) Tomographic reconstruction of internal wave patterns in a paraboloid. Exp Fluids 50(2):247-258

Horne E et al (2019) Particle transport induced by internal wave beam streaming in lateral boundary layers. J Fluid Mech 870:848-869

Ibrahim RA (2005) Liquid sloshing dynamics: theory and applications. Cambridge University Press, Cambridge

Jouve L, Ogilvie GI (2014) Direct numerical simulations of an inertial wave attractor in linear and nonlinear regimes. J Fluid Mech 745:223-250

Lam F-PA, Maas LRM (2008) Internal wave focusing revisited: a reanalysis and new theoretical links. Fluid Dyn Res 40(2):95

Lenci M, Bonanno C, Cristadoro G (2021) Internalwave billiards in trapezoids and similar tables. arXiv:2102.01654

Maas LRM et al (1997) Observation of an internal wave attractor in a confined, stably stratified fluid. Nature 388(6642):557561

Maas LRM (2001) Wave focusing and ensuing mean flow due to symmetry breaking in rotating fluids. J Fluid Mech 437:13

Maas LRM (2005) Wave attractors: linear yet nonlinear. Int J Bifurc Chaos 15(09):2757-2782

Maas LRM, Lam F-PA (1995) Geometric focusing of internal waves. J Fluid Mech 300:1-42

Manders AMM, Maas LRM (2003) Observations of inertial waves in a rectangular basin with one sloping boundary. J Fluid Mech 493:59 
Mowbray D, Rarity B (1967) A theoretical and experimental investigation of the phase configuration of internal waves of small amplitude in a density stratified liquid. J Fluid Mech 28(1):1-16

Pillet $\mathrm{G}$ et al (2018) Internal wave attractors in three dimensional geometries: trapping by oblique reflection. J Fluid Mech 845:203

Scolan H, Ermanyuk E, Dauxois T (2013) Non linear fate of internal wave attractors. Phys Rev Lett 110(23):234501

Tian X et al (2017) Hydrodynamic coefficients of oscillating at plates at 0:15 le KC le 3:15. J Mar Sci Technol 22(1):101113

van Oers AM, Maas LRM, Bokhove O (2017) Hamiltonian discontinuous Galerkin FEM for linear, stratified (in)compressible Euler equations: internal gravity waves. J Comput Phys 330:770-793
Voisin B, Ermanyuk EV, Flóor J-B (2011) Internal wave generation by oscillation of a sphere, with application to internal tides. J Fluid Mech 666:308-357

Publisher's Note Springer Nature remains neutral with regard to jurisdictional claims in published maps and institutional affiliations. 